\title{
Litigare in tragedia: per una pragmatica del conflitto*
}

\author{
Federica Iurescia \\ Universität Zürich \\ federica.iurescia@sglp.uzh.ch \\ ORCID iD: https://orcid.org/0000-0001-5100-5539
}

\section{Quarrels in Roman tragedy: impoliteness in conflict}

\begin{abstract}
Il presente lavoro ha come oggetto un tipo di conversazione, la lite, in un genere letterario, la tragedia romana, e ne offre un'analisi pragmatica. Partendo da una definizione del tipo di conversazione oggetto di studio, si applica alle attestazioni reperite nel corpus scelto l'approccio maturato entro il settore degli studi sull'im/politeness. Le osservazioni risultanti vengono messe a confronto con le tendenze rilevate in un precedente studio sulle liti nella commedia e nel romanzo latini, mettendo in luce convergenze e differenze.
\end{abstract}

Parole chiave: Seneca; Tragedia; lite; pragmatica; impoliteness.
This paper offers a pragmatic analysis of quarrels in Roman Tragedy. Its aim is to study this kind of conflictual interactions from a pragmatic perspective, and to compare tragic quarrels with quarrels in Roman Comedy and Novel, object of a previous study. The theoretical approach situated in the realm of im/politeness theories allows to describe the linguistic forms used in quarrels, and provides a coherent framework to study impoliteness in Latin, delineating patterns of behaviour through different literary genres across a wide time span. This paper offers a further step in this direction.

Key words: Seneca; Tragedy; Quarrel; Pragmatics; Impoliteness.

Cómo citar este artículo / Citation: Iurescia, Federica 2019: «Litigare in tragedia: per una pragmatica del conflitto», Emerita 87 (2), pp. 255-283.

\section{INTRODUZIONE}

Questo lavoro si pone in linea di continuità con l'analisi pragmatica delle liti nelle fonti letterarie latine svolta in Iurescia 2019; ne condivide la

* Questo articolo è stato scritto nell'ambito del progetto di ricerca SNF Die Pragmatik des Dialogs in der antiken Tragödie. PNr. PP00P1_157444 / 1. 
metodologia -un approccio basato sugli studi sull'impoliteness elaborati principalmente da Jonathan Culpeper (Culpeper 1996; 2011; 2016) ${ }^{1}$ - e ne integra il corpus, affiancando alla ricerca sulla commedia e sul romanzo l'indagine su un altro genere letterario, la tragedia. Il corpus tragico è omogeneo per genere e cronologia, ma esiguo: ci sono pervenute dieci opere per intero ${ }^{2}-$ le otto opere di Seneca e le due di autori incerti, Hercules Oetaeus e Octavia, tutte databili all'incirca al I d. C.- e al loro interno si sono individuati cinque esempi di lite, tutti nelle tragedie di Seneca. Si tratta degli scontri tra Clitennestra ed Elettra, madre e figlia, nell'Agamemnon ai versi 953-980; ai versi 981-1000 tra Elettra ed Egisto, l'amante di Clitennestra e complice nell'assassinio di Agamennone; tra Medea e Giasone, ex marito e moglie (Sen., Med. 445-559); tra i guerrieri Agamennone e Pirro nelle Troades ai versi 203-352; tra Lico, usurpatore del trono di Ercole e Megara, moglie di Ercole, nell'Hercules Furens (Sen., Herc. F. 359-438; 491-515) ${ }^{3}$.

Inizio ricordando la definizione di lite che ho proposto per individuare il tipo di conversazione oggetto d'analisi: per lite si intende una comunicazione conflittuale tra almeno due parlanti che mostrino aggressività reciproca. In tutti e cinque i casi del corpus tragico si tratta di liti rappresentate, per cui si possono ricavare informazioni sulla struttura della conversazione -sulle fasi di apertura e chiusura, che spesso risultano realizzate in modo non standarde sulle forme linguistiche impiegate dai parlanti per esprimere la loro aggressività. Protagonisti sono personaggi di alto statuto ${ }^{4}$, legati da stretti rapporti (Medea, Agamemnon) o che comunque si conoscono bene (Troades, Hercules Furens).

${ }^{1}$ Per una trattazione dettagliata v. Iurescia 2019, pp. 17-25. In tal senso il lavoro si pone nell'ambito della ricerca sull'im/politeness in Latino, un settore in continua espansione: per una rassegna sullo stato degli studi v. Unceta Gómez 2018.

${ }^{2}$ Nei frammenti delle tragedie non si è rinvenuto materiale utile all'analisi.

${ }^{3}$ Le tragedie sono elencate nell'ordine cronologico di composizione proposto da Fitch 1981. Per quanto riguarda la rappresentazione delle liti non si riscontrano differenze significative tra i vari casi, che appaiono equamente distribuiti nell'arco della produzione drammatica.

${ }^{4}$ Com'era da aspettarsi, dato che nelle tragedie a noi pervenute per intero gli unici personaggi di statuto inferiore a dèi, indovini, membri di famiglie regali e condottieri sono messaggeri, nutrici, accoliti, il pastore nell'Oedipus e l'araldo nelle Troades. 


\section{ANALISI DELLE SCENE DI LITE}

1. Openings e Closings: Sen., Tro. 203-352

Cominciamo con la lite nelle Troades, che ci introduce nella problematica della realizzazione dell'apertura di una conversazione conflittuale. A scontrarsi in questo caso sono Agamennone, comandante dell'armata greca nella guerra di Troia, e Pirro, greco anche lui e figlio di Achille. Siamo agli atti finali della guerra; dopo la morte del padre, Pirro ha raggiunto Troia per unirsi all'esercito greco e rivendica gli onori dovuti e non resi alla memoria di Achille. In particolare, nella lite in questione, Pirro richiede il sacrificio della principessa troiana Polissena, figlia di Priamo, da immolare sulla tomba di suo padre. Agamennone invece si rifiuta di avallare questo sacrificio umano: questo l'antefatto della lite che Seneca rappresenta nelle fasi iniziali della tragedia $^{5}$. È proprio in questo modo che Pirro ed Agamennone entrano in scena; i due potrebbero essersi incontrati ed aver iniziato a parlare in precedenza ${ }^{7}$, cominciando l'interazione nel modo che è stato ricostruito come consueto per il latino (Hofmann 1983; Ferri 2008; Roesch 2008; Poccetti 2010). Trattandosi di una lite però, è anche possibile che l'inizio di conversazione non sia quello usuale in cui i parlanti innanzitutto si salutano, poi indugiano più o meno brevemente in small talks ed infine entrano nel merito della conversazione (Berger 2016). Maggiormente conforme al tipo di comunicazione in corso, sarebbe infatti saltare i convenevoli e passare direttamente a ciò che sta a cuore ai parlanti, in questo caso a Pirro, che parla così:

(1) Sen., Tro. 203-206

PY. Cum laeta pelago uela rediturus dares, excidit Achilles, cuius unius manu

${ }^{5}$ Sulla rielaborazione che Seneca compie del mito greco e sul rapporto con i modelli tragici greci molto si è scritto: nell'ampia bibliografia per lo stato della questione v. almeno Tarrant 1978; Dingel 1985; Goldberg 2014 con relativi rimandi bibliografici.

${ }^{6}$ Sulle possibili modalità di realizzazione scenica di questa entrata v. Fantham 1982, pp. 240-241.

${ }^{7}$ Così Keulen 2001, p. 195; Heil 2013, pp. 136-137.

${ }^{8}$ I testi latini delle tragedie sono citati secondo l'edizione di Zwierlein 1986; le traduzioni sono a mia cura. 
impulsa Troia, quidquid adiecit morae

illo remoto, dubia quo caderet stetit?

PI: Quando davi al mare le vele liete per il ritorno, è stato tagliato fuori Achille, per la cui sola mano

Troia sbaragliata, qualunque indugio abbia aggiunto

rimosso lui, è stata in dubbio su dove cadere?

Esempi di lite che inizino in modo altrettanto brusco si riscontrano nella commedia e nel romanzo'; per le tragedie di Seneca in quattro delle cinque liti è riportato l'inizio della comunicazione, ed in due casi sicuramente non si tratta di un'apertura standard. Nella Medea la protagonista apostrofa direttamente Giasone con accorati rimproveri e accuse (Sen., Med. 447-489); nell'Agamemnon (953-977) Clitennestra inizia ${ }^{10}$ insultando Elettra in modo vistoso (8). Non sembra quindi fuori luogo pensare ad un'apertura di conversazione anche in questo caso delle Troades, con Pirro che inizia con l'accusa ad Agamennone di non aver tributato i dovuti onori ad Achille. Un inizio brusco per una lite nelle tragedie di Seneca sarebbe quindi attestato in tre casi su cinque, o perlomeno sicuramente in due, quelli della Medea e dell'Agamemnon, in cui il testo consente di affermare con sicurezza che si tratti di apertura di conversazione; naturalmente, l'esiguo numero di esempi conferisce una forte relatività alle statistiche che se ne potrebbero trarre. Si deve inoltre notare che (1) è l'unico esempio in cui non vengono segnalati i movimenti in scena dei personaggi, al contrario degli altri casi.

Il giovane si dimostra da subito aggressivo: l'accusa ricorre nelle scene di lite rappresentate nella commedia e nel romanzo e coincide con una

${ }^{9}$ V. P1., Cas. 593-598; Men. 486-493; 707-710; Mos. 1-6; Rud. 492-493; Ter., Ad. 160161. Poiché in tutti questi casi ci sono indicazioni di movimenti sulla scena, non è possibile sulla base di tale confronto intervenire sulla discussione in merito alla possibile realizzazione scenica delle Troades in cui non ci sono indizi sui movimenti dei due personaggi. Per esempi analoghi di inizio brusco in una lite nel romanzo v. Petr. 14.5, 95.2-4; per un caso in cui, apparentemente, si passa subito alle mani senza parlare v. Apul., Met. VII 25. Per avere un'idea di quanto sia frequente questo tipo di apertura nelle liti si consideri che nel corpus costituito dalla commedia e dal romanzo (Iurescia 2019) si sono individuate trentasei rappresentazioni di lite; in ventitré di queste è testimoniata l'apertura della conversazione. In otto casi su ventitré quindi l'inizio è messo in scena in questo modo che viola le convenzioni: si tratta all'incirca del $35 \%$.

${ }^{10}$ Il suo rientro in scena è descritto da Elettra stessa subito prima: Sen., Ag. 947-952. 
delle forme di impoliteness riconosciute da Culpeper ${ }^{11}$. L'intervento di Pirro, piuttosto lungo (203-249), è seguito da una replica altrettanto lunga di Agamennone (250-291) ${ }^{12}$, dopo la quale gli interventi dei due diventano progressivamente più brevi fino ad arrivare alla sticomitia (327-336), per poi distendersi nuovamente fino all'incirca ai due versi per ciascuno (337351). Le ripetute accuse (203-249), insulti (301-303) e minacce (306-310) da parte di Pirro portano Agamennone a cambiare atteggiamento. All'inizio valuta le parole del suo interlocutore come un difetto (uitium 250) insieme personale, dovuto alla sua giovane età, ed ereditato dal padre (250-252), ed imprime un forte tono sentenzioso alla sua replica (254-291), senza manifestare forme di impoliteness. Dopo gli attacchi ricevuti invece abbandona le sentenze ed inasprisce l'offesa personale adombrata in quel suo primo riferimento al uitium. Siamo qui nella lite vera e propria: Agamennone diventa scopertamente aggressivo con l'ironia con cui rinfaccia a Pirro l'uccisione di Priamo, supplice del padre (310-313), e l'atteggiamento indifferente di Achille in mezzo alla strage dei Greci (318-321); quando Pirro risponde colpo su colpo (313-317, 322-324), Agamennone ripete il suo disprezzo per l'assassino del vecchio re (325-326). Pirro attacca in maniera diretta ed esplicita ${ }^{13}$; Agamennone si serve dell'ironia ${ }^{14}$ e prende le distanze

${ }_{11}$ «Explicitly associate the other with a negative aspect» Culpeper 1996, p. 358.

12 Per la struttura di questa conversazione v. Seidensticker 1969, pp. 163-179. Keulen 2001, p. 195 assimila questa prima parte dello scontro tra Agamennone e Pirro a due suasoriae; occorre però ricordare che la conflittualità codificata si serve di forme di impoliteness tipiche degli scontri non sorvegliati, epurandole in modo da accordarsi alla situazione comunicativa e allo status dei partecipanti - come il caso dell'altercatio ben esemplifica: v. Iurescia 2019, p. 144.

${ }^{13}$ Si sono rilevate accuse (207-208); insulti -ove possibile, se ne riportano le parole-chiave (tumide ... timide ... regum tyranne 301-305; tu, graui pauidus metu 315-317, 322, 339)-; minacce (306-310); derisione $(331,340$, in cui Pirro viola anche le norme conversazionali che governano la gestione ordinata dei turni di parola, interrompendo Agamennone. Un'ulteriore interruzione, ma senza derisione, si riscontra al v. 344). Si noti che queste manifestazioni coincidono con alcune delle forme di impoliteness riconosciute da Culpeper. Sull'attribuzione all'interlocutore di caratteristiche negative si è già detto supra $\mathrm{n}$. 11; vengono menzionate anche pratiche come «Frighten - instill a belief that action detrimental to the other will occur.» (Culpeper 1996, p. 358), in cui si possono riconoscere le minacce, e «Condescend, scorn or ridicule» (ibidem) descrizione che si adatta bene alla derisione che più volte punteggia la scena.

${ }^{14}$ Forme ironiche con cui Agamennone riveste i propri insulti a Pirro occorrono ai versi 310-313, 318-321, 325-326, 328, 330, 347. Ironia sembra permeare l'intervento di Pirro ai 
dal proprio interlocutore parlando di lui in terza persona, come se non fosse lì (Haud equidem nego / hoc esse Pyrrhi maximum in bello decus, / saeuo peremptus ense quod Priamus iacet, / supplex paternus. 310-313 $)^{15}$ : si tratta della non-interpellation, un espediente linguistico che, tra le funzioni che può svolgere, vale anche a veicolare attacchi in contesti aggressivi (Orlandini, Poccetti 2010).

Lo scambio serrato della sticomitia configura un fuoco di fila di accuse in cui Agamennone prima si muove sul piano dell'offesa personale $(328,330)$ e poi passa ad esprimersi su un piano più generale, difendendo la propria condotta con frasi di sapore sentenzioso $(332,334,336)$, cui Pirro risponde a tono con frasi di carattere altrettanto generale $(327,329,333,335)^{16}$, per poi tornare sul piano dell'attacco personale (337-338) su cui trascina anche Agamennone ${ }^{17}$ :

(2) Sen., Tro. 337-348

PY. His ista iactas, quos decem annorum graui

regno subactos Pyrrhus exsoluet iugo?

AG. Hos Scyros animos? PY. Scelere quae fratrum caret.

AG. Inclusa fluctu-PY. Nempe cognati maris:

Atrei et Thyestae nobilem noui domum.

AG. Ex uirginis concepte furtiuo stupro

et ex Achille nate, sed nondum uiro-

PY. Illo ex Achille, genere qui mundum suo,

sparsus per omne caelitum regnum, tenet:

versi 248-249. Per una discussione sull'ironia dalla prospettiva dell'impoliteness v. Culpeper 1996, pp. 356-357; Culpeper 2011, pp. 165-180.

${ }_{15}$ «Non nego, certo, / che questo sia il massimo onore in guerra per Pirro, / che Priamo, supplice di suo padre, giaccia ucciso / da una spada selvaggia.»

${ }^{16}$ Sullo scambio di sententiae ai versi 333-336 da parte di entrambi gli interlocutori v. Seidensticker 1969, p. 169; Casamento 1999, pp. 128-129; Calabrese 2009, pp. 93-94. Già Traina $2011^{4}$ [1974], p. 25 notava «... certo la cellula stilistica di Seneca e della sua età è la frase, la sententia; nell'epoca di Cesare e di Cicerone era stato il periodo; nell'epoca di Frontone sarà la parola.» Per una raccolta di sententiae nelle tragedie di Seneca v. Kunz 1897; Canter 1925, pp. 85-99.

17 Agamennone attribuisce ora a Pirro caratteristiche estremamente negative senza schermarle con l'ironia con cui lo aveva insultato in precedenza $(339,342-343$, v. 340 su Sciro, la patria di Pirro ed Achille). 
Thetide aequor, umbras Aeaco, caelum Ioue.

AG. Illo ex Achille, qui manu Paridis iacet.

PY. Quem nec deorum comminus quisquam petit.

PI: Queste cose le vai a dire a questi, sottomessi per dieci anni ad un comando gravoso, che Pirro libererà dal giogo?

AG: Quest'arroganza Sciro? PI: Che manca di scelleratezze tra fratelli.

AG: Chiusa tra le onde-PI: Certo di un mare familiare:

ho conosciuto la nobile casata di Atreo e Tieste.

AG: Concepito dallo stupro furtivo di una vergine

nato sì da Achille, ma non ancora uomo-

PI: Da quell'Achille che tiene il mondo con la sua stirpe,

diramato in ognuno dei regni dei celesti:

il mare da Teti, le ombre da Eaco, il cielo da Giove.

AG: Da quell'Achille che giace per mano di Paride.

PI: Che nessuno degli dei ha voluto a duello.

A questo punto Agamennone tronca la conversazione: si ritrae evitando di rivolgersi direttamente al proprio interlocutore e si rifugia ancora una volta in un'espressione dal carattere generale:

(3) Sen., Tro. 349-352

AG. Compescere equidem uerba et audacem malo

poteram domare; sed meus captis quoque

scit parcere ensis. potius interpres deum

Calchas uocetur: fata si poscent, dabo.

AG: Certo avrei potuto tenere a freno le parole e domare

con un castigo uno sfrontato; ma la mia spada sa risparmiare

anche i prigionieri. Piuttosto sia chiamato l'interprete degli dei,

Calcante: se sarà il fato a richiedere, darò.

Agamennone introduce in questo modo la chiusura della conversazione ${ }^{18}$ e chiama Calcante: saranno i fata (352) a dirimere la questione che ha dato inizio alla lite. Con un gesto arbitrario quindi viene troncata una conversa-

${ }^{18}$ Sulle chiusure di conversazioni conflittuali nelle tragedie greche e latine v. Iurescia, Martin (2019). 
zione che dal motivo della lite era scivolata sul piano delle accuse personali, per di più ripetendole, incanalandosi così in un vicolo cieco ${ }^{19}$ : tale dinamica si ritrova anche in altri passi delle tragedie di Seneca ${ }^{20}$, sempre in casi in cui il parlante sembra a corto di argomenti da opporre al proprio interlocutore e decide quindi di sottrarsi al confronto chiudendo l'interazione. In altre parole, quando chi parla è a corto di argomenti, una delle reazioni possibili è la chiusura forzata della conversazione. Pirro con i suoi ripetuti attacchi causa in Agamennone repliche aggressive gradualmente sempre più dirette; tale reazione suggerisce che quegli attacchi, e verosimilmente anche l'insistenza con cui vengono reiterati, siano stati percepiti come offensivi. Carenti nella scena sono le notazioni di carattere metapragmatico: solo alla fine, quando Agamennone introduce la chiusura esprime la propria percezione dell'interazione, descrivendo l'atteggiamento di Pirro come $a u$ dacem $^{21}$.

La scena funge da presentazione dei caratteri dei due personaggi ${ }^{22}$, e da focalizzatore su un tema critico -il sacrificio di Polissena- che era già stato introdotto: la lite quindi non contribuisce alla progressione dell'intreccio, piuttosto ne pone in rilievo una fase problematica ${ }^{23}$. Queste caratteristiche sul piano narratologico erano emerse anche nella trattazione delle liti rappresentate nella commedia e nel romanzo; la lite delle Troades si pone quindi in linea di continuità con quanto si era potuto osservare in generi letterari diversi. Proseguiamo la rassegna del corpus tragico con la Medea, in cui, come si è anticipato, l'interazione viene rappresentata fin dall'apertura.

19 Sulla ripetitività di una conversazione che finisce in una strada senza uscita v. Seidensticker 1969, pp. 78-79; la circolarità di questo passo delle Troades è sottolineata nei commenti di Fantham 1982 ad l. e Keulen 2001 ad l., senza tuttavia esaminarla da un punto di vista pragmatico. V. anche Speyer 2003, pp. 92-93.

${ }^{20}$ Ag. 800-801, 978-980 (9); 997-1001 (11), Oed. 707; si confronti Med. 530 in cui Giasone, nel corso della sua lite con Medea, tenta di troncare la conversazione (infra pp. 264-265).

${ }^{21}$ La mancanza di senso della misura è la nozione denotata da audax, dove il suffisso - ax è indice di una modalità intensa e ripetuta dell'azione espressa dal verbo: v. Leumann $1977^{5}$ [1926], p. 376. Per uno studio diacronico sugli aggettivi latini in -ax v. de Nigris Mores 1972. Cf. l'analoga nozione, rafforzata dal prefisso per- in pertinax e peruicax (così Lico descrive Megara che si rifiuta di sposarlo: Sen., Herc. F. 493 (6) e 501 (7) rispettivamente).

${ }^{22}$ Così già Steidle 1941, p. 280.

${ }^{23}$ V. Heil 2013, pp. 161-162. 
2. Iniziare con una lite, finire con un inganno: Sen., Med. 447-489

La prima occasione in cui Giasone e Medea si trovano faccia a faccia nell'omonima tragedia di Seneca si articola in una lite: Giasone ha fatto da poco la sua entrata in scena (Sen., Med. 431) commiserando la propria sorte infelice ed annunciando le proprie intenzioni nei confronti di Medea, che immagina in preda alla collera: le si rivolgerà con preghiere (constituit animus precibus iratam aggredi 444). Ma ecco comparire Medea, che -almeno stando al giudizio di Giasone- reca segni evidenti della propria ira; una conferma di tale interpretazione si può leggere nel modo in cui la donna comincia l'interazione, sostituendo le fasi iniziali tipiche di una conversazione standard con un esordio ex abrupto conforme ad uno stato d'animo alterato:

(4) Sen., Med. 447-450

IA. atque ecce, uiso memet exiluit, furit, fert odia prae se: totus in uultu est dolor.

ME. Fugimus, Iason, fugimus - hoc non est nouum, mutare sedes; causa fugiendi noua est: pro te solebam fugere-discedo, exeo, penatibus profugere quam cogis tuis.

GIA: Ma ecco, sobbalza al vedermi, una furia, l'odio è evidente: tutto il suo dolore sul volto.

ME: Sono fuggita, Giasone, fuggo-questo non è nuovo, cambiare sede; nuovo è il motivo della fuga: ero solita fuggire per te-mi ritiro, me ne vado, io che tu costringi a fuggire dai tuoi penati.

L'aspetto (uultus) ${ }^{24}$ di Medea, i suoi movimenti (exiluit) ${ }^{25}$ ed il suo atteggiamento verbale concordano quindi nel contrassegnare un approccio marcato in senso ostile: accuse $(450,458,459-460,483-489)$, ironia (451-453,

${ }^{24}$ Sul uultus come espressione dell'interiorità v. Bettini 2000, in particolare alle pp. 323-336.

${ }^{25} \mathrm{~V}$. ThLL 5.2.1865.54, dove si motiva il movimento improvviso con l'insorgere di una forte emozione. 
454-456), alternanza tra allocuzione diretta e non-interpellation ${ }^{26}$ (exuli exilium imperas / nec das. eatur. regius iussit gener 459-460), appellativi non pertinenti alla loro relazione ${ }^{27}$ (regius ... gener 460), insulti (adulter 456; ingratum $^{28}$ caput 465 ) esprimono chiaramente l'aggressività della donna verso Giasone. Inizialmente questi non replica agli attacchi ricevuti e prova prima a guadagnarsi riconoscenza per averle salvato la vita, essendosi impegnato personalmente per farle avere l'esilio invece della pena di morte (490491); dato che la mossa si rivela inefficace (492), cerca di persuaderla ad andare via agitando lo spauracchio delle ritorsioni di chi ha potere, in questo caso Creonte, contro Medea che, esule e sola, si troverebbe in una condizione di debolezza (493-494). Neppure stavolta Medea si lascia convincere: il secondo fine di Giasone è in realtà quello di allontanarla a guadagno di Creusa, la sua promessa sposa nonché figlia di Creonte (494-495). È a questo punto che Giasone cambia atteggiamento: tramite accuse formulate per via di domande retoriche $(496,497)$ e asserzioni con cui si dissocia dai misfatti di Medea (498-499) ${ }^{29}$, passa ad esprimersi anche lui con delle forme di impoliteness $^{30}$. Medea insiste sull'accusa di colpevolezza: tutti i crimini che ha commesso sono stati compiuti per lui, che ne è quindi complice e corresponsabile (497-503); Giasone al contrario contesta questa presentazione dei fatti ed elenca le difficoltà e i pericoli cui andrebbero incontro, loro ed i loro figli, nel caso di una fuga insieme, come Medea vorrebbe (506-529). Questo atteggiamento non nasce dalla paura del potere di Creonte, ma dalla brama di conquistarlo, obietta Medea (529): di fronte a questa ennesima accusa, Giasone cerca di troncare la conversazione (suspecta ne sint, longa colloquia amputa 530). Preparando la chiusura e presentandone la motivazione (su-

\footnotetext{
${ }^{26}$ Specificamente su questo passo della Medea v. Orlandini, Poccetti 2010, §§ 20-21.

${ }^{27}$ Anche questa riconosciuta come una forma di impoliteness: «Use inappropriate identity markers -for example, use title and surname when a close relationship pertains, or a nickname when a distant relationship pertains.» Culpeper 1996, p. 357.

${ }^{28}$ Sull'ingratitudine di Giasone da un punto di vista antropologico v. Scolari 2018, pp. 267-275 cui rimando per ulteriore bibliografia sul tema.

${ }^{29}$ V. Culpeper 1996, p. 357: «Disassociate from the other -for example, deny association or common ground with the other; avoid sitting together. "

${ }^{30}$ Ve ne si può aggiungere anche un'altra, un insulto formulato in maniera obliqua con cui peraltro Giasone ritorce contro Medea quell'ingratitudine che lei (Sen., Med. 465) gli aveva rinfacciato: Ingrata uita est cuius acceptae pudet. «Sgradita è la vita a chi si vergogna di averla ricevuta» (504).
} 
specta ne sint), Giasone cerca di uscire dal circolo chiuso in cui il suo dialogo con Medea si è trasformato: dall'intenzione iniziale di rivolgerle delle preghiere (444) è passato ai tentativi di persuasione con diverse argomentazioni, sia che vertono maggiormente sulla razionalità («ti ho salvato la vita» 490-491), sia che fanno leva più scopertamente sulle emozioni, in questo caso paura (493-494) ed infine all'aggressività (496, 497, 498-499, 504), per poi virare sulla manipolazione per mezzo dei figli (506-507) ed approdare alle preghiere (513-514). Tutto inutile: Medea ribatte ogni volta, neppure gli ultimi tentativi con la paura delle conseguenze $(516,519,521,525-526)$ hanno presa su di lei. A Giasone sembra non rimanga nient'altro che chiudere il contraddittorio, ma Medea non glielo permette: alla proposta di terminare la conversazione reagisce cambiando interlocutore e pregando Giove di rivolgere i suoi fulmini su di loro (531-539) $)^{31}$. Questo non è l'atteggiamento atteso in una conversazione a due, per di più se il terzo invocato non è presente, è una divinità, e tantomeno se gli si chiede di annientare i parlanti: l'accumulo di questi elementi porta Giasone a valutare come pazzia l'atteggiamento di Medea e ad invitarla a più miti consigli (537-539). Medea sembra acconsentire, chiede di poter portare con sé i loro figli (540-543) e dalla replica di Giasone (544-549) scopre che è questo il suo punto debole; immediatamente decide di sfruttarlo per ferirlo nel modo più doloroso possibile (549-550) e si mostra quindi accondiscendente, anzi, lo ringrazia e chiede addirittura perdono (553-557). Che questo atteggiamento sia solo una finzione risulta evidente dalle parole che Medea rimasta sola pronuncia (560-578).

Medea, irata, apre l'interazione in modo aggressivo e continua così finché Giasone dichiara il proprio amore per i loro figli: la scoperta del mezzo più atroce per vendicarsi detta il cambiamento verso un comportamento marcato da forme di interesse per l'altro ${ }^{32}$. Diverso il comportamento linguistico di Giasone, che, conformemente all'intento dichiarato prima che l'interazione cominci, cerca di astenersi da forme aggressive e vi si lascia andare solo dopo che altri tentativi di persuasione si sono rivelati fallimentari, e solo per un bre-

31 V. Costa 1989 ad 530.

32 In questo senso nella conversazione si determina un punto importante per gli avvenimenti successivi, l'identificazione del modo di procedere per realizzare l'intento che anima la protagonista. Si noti però che quando questo avviene l'aggressività verbale lascia il posto a tentativi di riconciliazione: in altre parole, finché la lite sussiste, l'intreccio non va avanti. Sulla funzionalizzazione della lite nelle tragedie da un punto di vista narratologico v. infra III. 
ve tratto. Anche sotto questo aspetto quindi Medea, donna e straniera, viene rappresentata in stridente contrasto con un uomo non barbaro, quale Giasone è. Malgrado entrambi siano di status alto, questo dato comune rimane in ombra nella caratterizzazione, che gioca molto di più sulle differenze tra i due, ad evidenziare a tutto tondo come la loro identità di coppia sia improponibile.

\section{Persuasione all'inizio, lite alla fine: Sen., Herc. F. 359-438; 491-515}

Una dinamica simile, ai confini tra lite e persuasione, si riscontra anche nell'Hercules Furens. Siamo nella fase iniziale della tragedia: in assenza di Ercole, impegnato nella cattura di Cerbero per compiere una delle fatiche che il re Euristeo gli aveva comandato, il governo di Tebe è stato usurpato dall'esule Lico, il quale, per legittimare il proprio potere, mira a sposare Megara, moglie di Ercole. Lico entra in scena al verso 33233; nel monologo che segue esprime la necessità di rendere stabile il dominio che ha conquistato con il proprio valore tramite quel che gli manca e non può ottenere da solo: la parentela con una nobile stirpe, che egli vuole acquisire attraverso il matrimonio con Megara (338357). Le si rivolge quindi in modo cerimonioso con un'allocuzione onorifica e chiedendole ascolto per un poco ( $O$ clarum trahens / a stirpe nomen regia, facilis mea / parumper aure uerba patienti excipe. Sen., Herc. F. 359-361). Dopo quest'esordio contrassegnato da forme di politeness ${ }^{34}$ passa a proporle un'unione che incontra il netto rifiuto della sposa di Ercole: non vuole avere niente a che fare con un assassino e un usurpatore, superbo tiranno (372-396). Il comportamento di Megara stride con quello di Lico: segnali non verbali - quid truci uultu siles? 'Perché taci con volto truce?' le chiede Lico al v. 371- e verbali-le accuse dei versi 379-380, l'associazione di Lico ad aspetti negativi e gli

33 Il suo arrivo è annunciato da Megara: Sed ecce saeuus ac minas uultu gerens / et qualis animo est talis incessu uenit / aliena dextra sceptra concutiens Lycus «Ma ecco, feroce e con segni di minaccia sul viso / e come l'animo, tale il passo, così avanza / brandendo nella destra lo scettro di un altro, Lico» (Sen., Herc. F. 329-331)

${ }^{34}$ L'allocuzione contiene elementi che sottolineano il valore dell'interlocutrice (positive face) e la richiesta di ascolto comunica l'intento di non volerne impedire la libertà (negative face). Questi due aspetti, la cui formulazione risale a Brown, Levinson 19872[1978], p. 62, e che si basano sugli studi di Goffman 1967, sono stati ripresi più e più volte nella ricerca sull'im/politeness. Per ragioni di spazio mi limito qui a segnalare per il latino nuovamente il contributo di Ferri 2008, in cui ci si concentra sull'apertura di conversazione. 
insulti (384-396)- manifestano l'ostilità della donna, che Lico esplicitamente riconosce, associandola alla collera: Agedum efferatas rabida uoces amoue «Su, lascia da parte rabbiosa voci efferate» (397). La invita quindi a sottostare alla propria autorità di re, e indulge a giustificare brevemente come necessità di guerra le azioni che lei gli ha rinfacciato (397-408); indi ripete l'invito a deporre l'odio, già menzionato (362-369) come primo argomento di persuasione e formula in maniera stavolta esplicita la proposta di matrimonio ${ }^{35}$. Megara è atterrita; si rifiuta, e lo fa in un modo particolare: non a Lico, ma in apostrofe ad Ercole assente si rivolge, per dichiarare che mai cederà all'usurpatore (Grauent catenae corpus et longa fame / mors protrahatur lenta: non uincet fidem / uis ulla nostram; moriar, Alcide, tua. 419-420). Nonostante l'interlocutore designato sia Ercole, il messaggio è indirizzato a Lico, in una forma di comunicazione a carambola ${ }^{36}$, che come tale viene decodificata da Lico, il quale infatti replica rivolgendosi direttamente a Megara (422). Comincia così uno scambio più serrato, in cui gli interlocutori abbandonano le frasi lunghe che avevano caratterizzato i loro interventi precedenti per passare alla sticomitia. Qui a mostrare aggressività è solo Lico, verosimilmente perché frustrato per gli ostinati rifiuti di Megara che in questo modo manderebbe a monte i suoi piani ${ }^{37}$ :

(5) Sen., Herc. F. 422-429

LY. Animosne mersus inferis coniunx facit?

ME. Inferna tetigit, posset ut supera assequi.

LY. Telluris illum pondus immensae premit.

ME. Nullo premetur onere, qui caelum tulit.

LY. Cogere. ME. Cogi qui potest nescit mori.

LY. Effare thalamis quod nouis potius parem

regale munus. ME. Aut tuam mortem aut meam.

LY. Moriere demens. ME. Coniugi occurram meo.

35 Sociemus toros (Sen., Herc. F. 413), più diretto del precedente sociemur animis; pignus hoc fidei cape (370).

${ }^{36} \mathrm{Si}$ tratta di una comunicazione obliqua, per così dire, in cui un parlante mira a comunicare un messaggio non al suo diretto interlocutore, ma ad un terzo destinatario: v. Wollams, Brown 1979; per il latino Ricottilli 2013. Cf. Kerbrat-Orecchioni 1997, pp. 4-5, la quale rimanda ai propri studi su questo meccanismo linguistico che definisce trope communicationnel.

37 V. Seidensticker 1969, pp. 76-77, il quale nota come il discorso finisca in un circolo, e nota le vittorie di Megara ad ogni replica. 
LI: Uno sposo immerso negli inferi ti dà questo ardire?

ME: Ha toccato l'inferno per poter raggiungere il cielo.

LI: Il peso della terra immensa lo schiaccia.

ME: Non viene schiacciato da nessun peso chi ha retto la volta del cielo.

LI: Sarai costretta. ME: Può essere costretto chi non sa morire.

LI: Dì piuttosto quale dono regale io possa offrire a pari

delle nuove nozze. ME: O la tua morte o la mia.

LI: Morirai, folle. ME: Andrò incontro al mio sposo.

Dopo la prima minaccia (426) Lico fa seguire un tentativo di conciliazione con la proposta di un dono (427-428); alla provocatoria replica di Megara oppone una nuova minaccia ed un insulto, segnale che la sua irritazione sta crescendo. La sposa di Ercole non si lascia intimidire, anzi: Coniugi occurram meo (429) è la sua replica, dalla quale Lico trae spunto per un confronto tra sé ed Ercole cercando di mettere il suo rivale in cattiva luce fino a porre in dubbio la sua ascendenza divina (430-438), punto in cui è Anfitrione, padre dell'eroe, ad intervenire, prendendo il posto di Megara come interlocutore, quale persona più adatta a garantire i natali di Ercole (439-441). Comincia così una contrapposizione in cui Anfitrione sostituisce Megara nel difendere Ercole dalle denigrazioni di Lico, finché questi taglia corto con una formulazione a carambola destinata a Megara:

(6) Sen., Herc. F. 489-500

... LY. Quod Ioui hoc regi licet:

Ioui dedisti coniugem, regi dabit;

et te magistro non nouum hoc discet nurus,

etiam uiro probante meliorem sequi.

sin copulari pertinax taedis negat,

uel ex coacta nobilem partum feram.

ME. Vmbrae Creontis et penates Labdaci

et nuptiales impii Oedipodae faces,

nunc solita nostro fata coniugio date.

nunc nunc, cruentae regis Aegypti nurus,

adeste multo sanguine infectae manus.

dest una numero Danais: explebo nefas.

... LI: Quel che è lecito a Giove, questo al re:

hai dato la sposa a Giove, lui la darà ad un re; 
e tua nuora imparerà questa cosa non nuova con te come maestro, a seguire uno migliore del marito, addirittura con la sua approvazione.

Se ostinata rifiuta l'unione con le fiaccole nuziali, avrò un parto nobile da lei dietro costrizione.

ME: Ombre di Creonte e Penati di Labdaco

e fiaccole nuziali dell'empio Edipo adesso date il solito destino al mio matrimonio.

Adesso, adesso, nuore cruente del re Egitto, accorrete, le mani infette di molto sangue.

manca una al numero delle Danaidi: io compirò il misfatto.

Come si vede, Megara riconosce l'intento illocutivo ed interviene, ancora una volta in modo particolare: continua a comunicare in maniera mediata, con un'apostrofe alle ombre negli Inferi il cui messaggio minaccioso è in realtà indirizzato a Lico. Questi ravvisa destinatario e messaggio e passa a rivolgerlesi direttamente, come si vede dai verbi alla seconda persona singolare (501-502). Al contempo però si distanzia da lei, ridefinendo i ruoli della relazione tra loro due: Megara, che ha rifiutato tutte le mosse di avvicinamento di Lico, e ha respinto il ruolo di sposa (Coniugia ... peruicax nostra abnuis 501), è adesso una sottoposta: Lico è il suo re, un re che lei ha appena cercato di spaventare (regemque terres 502). La conseguenza di questo atteggiamento è una punizione mortale:

(7) Sen., Herc. F. 501-508

LY. Coniugia quoniam peruicax nostra abnuis

regemque terres, sceptra quid possint scies.

complectere aras: nullus eripiet deus

te mihi, nec orbe si remolito queat

ad supera uictor numina Alcides uehi.

Congerite siluas: templa supplicibus suis

iniecta flagrent, coniugem et totum gregem

consumat unus igne subiecto rogus.

LI: Poiché caparbia rifiuti il matrimonio con me

e minacci il tuo re, saprai cosa possa uno scettro.

Abbraccia gli altari: nessun dio mi ti

strapperà, neppure se respinta la terra Alcide

vittorioso possa essere trasportato alle stelle del cielo. 
Accatastate legna: brucino i templi

attaccati ai loro supplici ed un unico rogo appiccato il fuoco consumi la sposa e tutto il seguito.

Alla minaccia segue il cambio di interlocutore con cui Lico si rivolge ai servi perché realizzino la punizione che egli ha previsto: considera chiuso il discorso e la questione, ma Anfitrione rilancia con la richiesta di morire per primo, richiesta alla quale Lico oppone senza pietà un netto rifiuto e annuncia la propria uscita, a confermare che la conversazione stavolta è davvero chiusa (509-515). La lite mette al centro due personaggi che altrimenti sono poco presenti: Lico compare solo qui, Megara era già in scena poco prima, in uno scambio preoccupato con Anfitrione sulle sorti di Ercole (309-331), e tornerà più tardi per morire (1008-1026), ma è attraverso questo scontro che si delineano i caratteri dei due personaggi.

Lico imposta la sua conversazione con Megara come un tentativo di persuasione: inizialmente è solo lei a manifestare aggressività, con forme di impoliteness che respingono con enfasi l'avvicinamento sperato da Lico. I reiterati rifiuti e il modo con cui sono formulati trascinano Lico per poco sul piano dell'aggressività (426-429); torna poi sulla persuasione cercando di mostrarsi migliore di Ercole, ma anche questa mossa viene respinta, sia da Megara sia da Anfitrione che subentra in quanto padre dell'eroe tanto denigrato a difendere i meriti del figlio, e impegna per un po' Lico su questo aspetto (439-489). Non con Anfitrione, ma con Megara Lico è però interessato a parlare, e torna a coinvolgerla nel modo prima mediato e poi diretto che si è descritto. I due si scambiano minacce (490-500) finché Lico, riconosciuto che ormai la sua proposta di matrimonio è tracimata in una lite senza uscita, chiude la conversazione.

\section{Litigare, litigare, litigare in tre: Sen., Ag. 953-980; 981-1000}

Chiaramente riconoscibile come una lite fin dall'inizio è invece l'interazione tra Clitennestra ed Elettra nell' Agamemnon $^{38}$, in cui i tratti di aggressività reciproca sono presenti dall'apertura alla chiusura del dialogo. Siamo nella fase finale della tragedia: il delitto è avvenuto, Elettra, comparsa sulla scena

${ }^{38}$ V. Seidensticker 1969, pp. 58-59. 
al v. 910, ha affidato il fratello Oreste a Strofio per sottrarlo al pericolo di essere assassinato anche lui dai due amanti. A questo punto vediamo rientrare in scena proprio Clitennestra che si rivolge alla figlia in modo esplicitamente aggressivo, insultandola (953) ed accusandola di tenere un comportamento indecente (954). Elettra da parte sua reciproca l'aggressività della madre (955) e questo atteggiamento si ripete per tutta la conversazione. In particolare, Elettra sembra riconoscerne e manipolarne le espressioni, come si vede già dal primo scambio (953-955), che ruota attorno alla parola uirgo. Clitennestra inizia il secondo sulla stessa linea, ma Elettra la interrompe completando la frase in modo che l'insulto a lei diretto si ritorca contro chi l'ha lanciato:

(8) Sen., Ag. 953-956

CL. Hostis parentis, impium atque audax caput, quo more coetus publicos uirgo petis?

EL. Adulterorum uirgo deserui domum.

CL. Quis esse credat uirginem-EL. Gnatam tuam?

CL: Nemica di chi ti ha generato, sacrilega e insolente, secondo quale costume tu, una vergine, ricerchi una pubblica assemblea? EL: Vergine, ho abbandonato una casa di adulteri.

CL: Chi crederà vergine-EL: Tua figlia?

Elettra riconosce l'insulto profilarsi nelle parole della madre, e ne previene la realizzazione compiuta interrompendola ${ }^{39}$; lo rispedisce poi al mittente attivando l'implicazione, evidente nelle proprie parole «chi nasce da te -che hai tradito ed assassinato tuo marito- non può essere considerata uirgo.» Il lemma continua quindi ad essere associato al complesso di attribuzioni per cui lo si era menzionato in precedenza e a valere come condensazione di un atteggiamento consono al proprio ruolo, ma con un repentino rovesciamento è proprio questo tipo di condotta rispettosa delle norme che la figlia nega alla madre. Clitennestra riprende il legame familiare contenuto nell'espressione

39 Anche l'interruzione è una violazione delle norme che regolano la struttura della conversazione -v. Levinson 1983, pp. 319-320-; sulla sua percezione però Clitennestra tace, come già Agamennone interrotto da Pirro (Sen., Tro. 340, 343-344) e non abbiamo quindi informazioni di carattere metapragmatico in proposito. 
della figlia (gnatam tuam) per innestarvi la propria replica: Modestius cum matre (957). Elettra riconosce un monito in queste parole, e ne seleziona l'intento (doces ibidem) esplicitandolo per offenderla: la materia che intende insegnare (pietatem 'contegno devoto' ibidem) non le compete per via dei misfatti che ha commesso. Clitennestra a questo punto riprende il giudizio negativo verso l'atteggiamento della figlia espandendo il quadro di riferimento: Elettra non solo non si comporta come una uirgo, ma neppure come una femina (958-959). Da parte sua la figlia è pronta a ritorcere contro la madre anche questa parola: Nisi forte fallor, feminas ferrum decet. 'Se non vado errata, la spada si addice ad una femmina' (960). L'espressione seguente della regina (964-966) rimane di non facile interpretazione: la prima parte (Indomita posthac uirginis uerba impiae / regina frangam; 964-965) potrebbe essere assimilabile ad un monologo ${ }^{40}$, mentre la seconda (citius interea mihi / edissere ubi sit gnatus, ubi frater tuus 965-966) rientra indubitabilmente nel dialogo, come dimostrano l'uso degli indicatori di seconda persona singolare $^{41}$ e la forza illocutiva di tipo direttivo del verbo. Per la prima parte però si può suggerire anche che si tratti di non-interpellation: Clitennestra, in altre parole, parla della figlia come se non fosse presente, e la insulta con la stessa parola ([uirginis] impiae) che in apertura le aveva rivolto direttamente (impium [caput] 953) ${ }^{42}$. Il rifiuto dello statuto pieno di interlocutore ed al contempo l'insulto indiretto valgono in questo caso a manifestare l'aggressività della regina, e proseguono l'atteggiamento comunicativo che ella ha te-

\footnotetext{
${ }^{40}$ Così Tarrant 1976 ad l.: «The words are spoken to no one in particular and are a kind of thinking out loud. »

${ }^{41}$ L'imperativo di seconda singolare edissere, e l'aggettivo possessivo tuus.

42 Ad ulteriore indizio della valenza dialogica anche di questa prima parte si può indicare la minaccia, pure questa veicolata indirettamente, espressa dal verbo frangam. Per quanto la si possa trovare anche in un monologo, nell'interpretazione come dialogo essa rientrerebbe, al pari dell'insulto, nella strategia di comunicare in maniera obliqua con la figlia: si tratterebbe di un modo indiretto di esprimere impoliteness. Ci si può chiedere se contenuti espressi in modo indiretto risultino più offensivi di quelli espressi in modo diretto: su questo tema, controverso anche negli studi di linguistica contemporanea, $i$ testi latini non sembrano fornire informazioni: v. Iurescia 2019, p. 43 n. 107. Sui commissivi, di cui la minaccia è un caso prototipico, v. Risselada 1993, p. 42.
} 
nuto fin dall'inizio. Ora però (965) subentra un nuovo intento ${ }^{43}$, direttivo stavolta: la richiesta di informazioni su dove sia Oreste ${ }^{44}$ :

(9) Sen., Ag. 964-980

CL. Indomita posthac uirginis uerba impiae

regina frangam; citius interea mihi

edissere ubi sit gnatus, ubi frater tuus.

EL. Extra Mycenas. CL. Redde nunc gnatum mihi.

EL. Et tu parentem redde. CL. Quo latitat loco?

EL. Tuto quietus, regna non metuens noua:

iustae parenti satis. CL. At iratae parum.

morieris hodie. EL. Dummodo hac moriar manu.

recedo $\mathrm{ab}$ aris. siue te iugulo iuuat

mersisse ferrum, praebeo iugulum tibi;

seu more pecudum colla resecari placet, intenta ceruix uulnus expectat tuum.

scelus paratum est: caede respersam uiri

atque obsoletam sanguine hoc dextram ablue.

CL. Consors pericli pariter ac regni mei,

Aegisthe, gradere. gnata genetricem impie

probris lacessit, occulit fratrem abditum.

CL: D'ora in avanti da regina schiaccerò le parole sfrenate

di un'empia vergine; nel frattempo veloce, veloce

riferiscimi dove sia mio figlio, dove tuo fratello.

EL: Fuori Micene. CL: Rendimi adesso mio figlio.

EL: E tu rendimi mio padre. CL: In che luogo si nasconde?

EL: Al sicuro, tranquillo, senza timore per nuovi regni:

abbastanza per una madre giusta. CL: Ma poco per una in collera.

Oggi morirai. EL: Purché muoia per questa mano.

Mi allontano dagli altari. Se ti piace in gola

aver immerso il ferro, porgo a te la gola;

${ }^{43}$ V. Tarrant 1976 ad 284, il quale osserva come il parlante in posizione debole spesso cerchi di cambiare discorso.

${ }^{44}$ Il modo in cui Clitennestra definisce Oreste è indicativo di come la regina percepisca e costruisca la propria identità: v. le osservazioni in proposito di Calabrese 2010, p. 147 e, più in generale su tutto il dialogo tra Clitennestra ed Elettra, ivi pp. 144-148. 
se mi vuoi sgozzata come le vittime sacrificali, la mia nuca protesa attende la tua ferita.

Il delitto è preparato: macchiata e insozzata dell'uccisione di tuo marito lava la tua destra con questo sangue.

$\mathrm{CL}$ : Tu che sei a parte del pericolo così come del mio regno, Egisto, avvicinati. Mia figlia empiamente tartassa con infamie chi l'ha generata, tiene segreto il fratello nascosto.

La lite quindi prosegue su questo nuovo tema: Elettra rifiuta di rivelare quello che la madre le chiede (967-970), e quando viene minacciata di morte (970-971) si dice pronta ed anzi disposta a morire (971-977). A questo punto la regina tronca la lite rivolgendosi ad Egisto e chiedendogli di intervenire (978-980). Nulla nei passaggi precedenti ha preparato questa mossa, l'introduzione non negoziata ed improvvisa di un terzo interlocutore. Nella percezione di Clitennestra la lite diventa una serie di infamie lanciatele contro dalla figlia (impie ${ }^{45}$ / probris lacessit, 979-980), senza tener conto delle forme di aggressività con cui lei stessa l'ha colpita, e senza menzionare che è stata proprio lei, Clitennestra, ad aprire l'interazione come lite, mentre Elettra ha solo risposto con impoliteness di reazione (Kienpointner 1997).

Egisto interviene prontamente, apostrofando Elettra a insulti e ordini:

(10) Sen., $A g$. 981-985

AE. Furibunda uirgo, uocis infandae sonum

et aure uerba indigna materna opprime.

EL. Etiam monebit sceleris infandi artifex, per scelera natus, nomen ambiguum suis, idem sororis gnatus et patris nepos?

${ }^{45}$ L'atteggiamento di Elettra viene percepito come negativo in quanto manchevole, come i composti col prefisso in- suggeriscono: (impie Sen., Ag. 979; è un'impia uirgo - 964 - che ha pronunciato indomita uerba - 963; anche Egisto lo descriverà in questi termini - uocis infandae sonum 981; uerba indigna 982). Del resto Clitennestra la vedeva così fin dall'inizio della loro interazione (impium atque audax caput 953, in cui il difetto di pietas e l'eccesso di baldanza in audax denotano entrambi una caratterizzazione negativa). Sulla percezione negativa di Elettra da parte di Clitennestra ed Egisto v. Paschalis 2010, pp. 223-224; Vandersmissen 2019, pp. 152-154. 
EG: Vergine folle, reprimi il suono

della tua voce infame e le parole indegne delle orecchie di tua madre.

EL: Moniti, addirittura, dall'artefice di un infame delitto,

nato tra i delitti, di nome incerto perfino ai suoi,

insieme figlio di sua sorella e nipote di suo padre?

Egli imposta fin da subito l'interazione in modo aggressivo, al quale Elettra reagisce producendosi a propria volta in insulti; a questo punto Clitennestra interviene parlando della figlia come se non fosse presente, ed addirittura la minaccia, sempre in questa maniera obliqua (fratrem reddat aut animam statim. 987 $)^{46}$. Anche Egisto adotta il modulo della non-interpellation: tutto il suo contributo (988-993), in cui descrive la punizione per quel silenzio ostinato è declinato in terza persona, quasi la destinataria non fosse la propria interlocutrice di poco prima, ancora lì presente. È Elettra a rivendicare il proprio statuto attivo nella conversazione rivolgendosi direttamente ad Egisto, che questa volta riprende il dialogo (994-996). Per poco però: dopo aver ribadito la propria condanna (996) si rivolge ai servi chiedendo loro di attuare la punizione:

(11) Sen., Ag. 994-1000

EL. Concede mortem. AE. Si recusares, darem:

rudis est tyrannus morte qui poenam exigit.

EL. Mortem aliquid ultra est? AE. Vita, si cupias mori.-

Abripite, famuli, monstrum et auectam procul

ultra Mycenas ultimo in regni angulo

${ }^{46}$ Sulle caratteristiche di una conversazione con tre partecipanti v. Kerbrat-Orecchioni 1997; per il caso dell'Agamemnon rilevanti in particolare le osservazioni sulle diverse categorie di ascoltatori ed osservatori: ivi pp. 3-11; Kerbrat-Orecchioni 2004, in particolare alle pp. 11-18, dove vengono riprese e discusse le analisi di Goffman 1981 sul ruolo degli attori in una conversazione. Si noti che ai versi 987-993 Clitennestra ed Egisto si alleano in una coalizione, il cui messaggio è in realtà rivolto ad Elettra, per la quale si può quindi suggerire che si tratti di una comunicazione a carambola. Questo passo sembra fornire attestazione di un real trilogue (Kerbrat-Orecchioni 1997, p. 9-10), a differenza dell'episodio di lite dell'Hercules furens, in cui pure ci sono tre partecipanti, ma i loro interventi si organizzano a coppie di dialoghi successivi, in cui Megara ed Anfitrione si alternano come interlocutori di Lico, significativamente punteggiati da forme di comunicazione a carambola, in cui viene coinvolto il partecipante che non è l'interlocutore selezionato fino a quel momento. 
uincite saeptam nocte tenebrosi specus, ut inquietam uirginem carcer domet.

EL: Concedimi la morte. EG: Se la rifiutassi, te la darei: inesperto è il tiranno che punisce con la morte.

EL: Più della morte c'è ancora qualcosa? EG: La vita, se brami di morire.

Portate via, servi, il mostro e trasportatala lontano

fuori Micene nell'angolo più remoto del regno

legatela circondata dalla notte di una spelonca buia, così che il carcere domi l'irrequieta vergine.

Di nuovo, anche qui il contributo di Egisto riguarda proprio l'interlocutrice con cui ha appena chiuso l'interazione, anche stavolta ribadendo il giudizio negativo (monstrum 997; inquietam uirginem 1000); Elettra invece in questa fase non gli si rivolge con delle forme di impoliteness. L'interazione tra Clitennestra ed Elettra dapprima ed Egisto poi risulta aggressiva fin dall'inizio, presenta forme dirette e mediate, fino alla conclusione finale. Si noti che dopo le parole di Egisto interviene ancora una volta Clitennestra, invocando una punizione su Cassandra, lì presente, la quale reagisce in un breve scambio con cui la tragedia si chiude (1001-1012). Le liti dell'Agamemnon confermano la caratterizzazione dei personaggi emersa nel corso della tragedia; ne costituiscono il finale conflittuale e luttuoso, che ben si situa a chiusura di un'opera in cui sangue, odio e vendetta hanno costituito le leve dell'azione scenica.

\section{LITIGARE IN TRAGEDIA: OSSERVAZIONI CONCLUSIVE}

Riassumiamo con l'aiuto di una tabella il comportamento linguistico che si è riscontrato nelle liti del corpus tragico; si noti che in molti casi le manifestazioni di aggressività vengono reciprocate o contraccambiate sotto altra forma. Quando questo non succede, si può individuare un motivo ben preciso, un secondo fine, che nei casi attestati coincide con la persuasione (Medea, Hercules Furens), o con la volontà di mostrare ritegno (Troades). Anche in questi casi comunque il parlante che inizialmente si era mantenuto su un piano neutro finisce per allontanarsene scivolando sul piano della lite. Questo tipo di reazioni consente di suggerire che alcune espressioni fossero valutate come aggressive; in alcuni casi-(3), (9) con n. 45, Herc. F. 397 supra p. 267-troviamo anche attestati dei commenti metapragmatici che confermano questa 
chiave di lettura. Indizi interni al testo e il confronto con scene di lite nella commedia e nel romanzo consentono di inquadrare tali atteggiamenti come forme di impoliteness, delle quali si presenta una panoramica in Tabella 1.

Tabella 1. Forme di impoliteness nelle liti delle tragedie di Seneca.

\begin{tabular}{|c|c|c|c|c|c|c|c|c|c|c|c|c|}
\hline Statuto & \multicolumn{12}{|c|}{ Forme verbali ${ }^{*}$} \\
\hline & \multicolumn{2}{|c|}{ Derisione } & \multicolumn{2}{|c|}{ Insulti } & \multicolumn{2}{|c|}{$\begin{array}{c}\text { Non- } \\
\text { interpellation }\end{array}$} & \multicolumn{2}{|c|}{$\begin{array}{l}\text { Violazione } \\
\text { struttura } \\
\text { conversazione }\end{array}$} & \multicolumn{2}{|c|}{$\begin{array}{l}\text { Appellativi } \\
\text { non pertinenti } \\
\text { a relazione }\end{array}$} & Minacce & Accuse \\
\hline & $\widehat{\mathrm{U}}^{* *}$ & $\sqrt{2}$ & $\hat{\imath}$ & $\sqrt{2}$ & iे & $\sqrt{2}$ & $\hat{v}$ & ת & $\hat{\imath}$ & $\sqrt{2}$ & & $\hat{\imath}$ \\
\hline Agamemnon & $\checkmark$ & $\checkmark$ & $\checkmark$ & $\checkmark$ & $\checkmark$ & & $\checkmark$ & $\checkmark$ & & & & $\checkmark$ \\
\hline Pyrrhus & $\checkmark$ & $\checkmark$ & $\checkmark$ & $\checkmark$ & & $\checkmark$ & $\checkmark$ & $\checkmark$ & & & & $\checkmark$ \\
\hline Iason & & & $\checkmark$ & $\checkmark$ & & $\checkmark$ & & $\checkmark$ & & $\checkmark$ & & $\checkmark$ \\
\hline Medea & & & $\checkmark$ & $\checkmark$ & $\checkmark$ & & $\checkmark$ & & $\checkmark$ & & & $\checkmark$ \\
\hline Megara & & & $\checkmark$ & & & & & & & & & \\
\hline Lycus & & & & $\checkmark$ & & & & & & & & $\checkmark$ \\
\hline Aegisthus & & & $\checkmark$ & $\checkmark$ & $\checkmark$ & & & & & & & \\
\hline Clytemestra & & & $\checkmark$ & $\checkmark$ & $\checkmark$ & & $\checkmark$ & $\checkmark$ & & & & \\
\hline Electra & & & $\checkmark$ & $\checkmark$ & & $\checkmark$ & $\checkmark$ & $\checkmark$ & & & & \\
\hline
\end{tabular}

* Il tratto della voce alta e forme non verbali non risultano attestati nel corpus esaminato.

** Il simbolo î indica chi compie l'atto in questione, ת a chi l'atto è indirizzato.

Come si vede, mancano completamente le forme di sconfinamento sul piano fisico: le manifestazioni di aggressività rimangono a livello verbale, conformemente con quella ripartizione del comportamento linguistico secondo l'asse sociologico (Iurescia 2019) per cui gli eccessi tendono ad essere associati a parlanti di statuto basso, quali non sono attestati tra i protagonisti delle liti del corpus tragico. Il contrasto, anche duro, con scambio di minacce ed insulti, non è mai sguaiato o chiassoso, come invece accade quando a litigare sono schiavi o il popolino. Le forme verbali più diffuse risultano insulti e accuse; le violazioni della struttura standard della conversazione riguardano per lo più la fase di chiusura; scarsamente attestate la 
non-interpellation e l'impiego di appellativi non pertinenti alla relazione. Non risultano chiare preferenze sulle modalità espressive associate al genere dei parlanti: le forme risultano equamente distribuite tra uomini e donne. Ribadendo che l'esiguità del corpus non consente di trarre conclusioni di carattere generale, si possono comunque affiancare alla conferma della tendenza di comportamento che si è appena rilevata alcune osservazioni. Partendo dalla realizzazione verbale notiamo l'assenza di informazioni su elementi paralinguistici: nulla viene detto sulle modalità vocali con cui si conduce la lite ${ }^{47}$, mentre nelle liti della commedia e del romanzo la voce alta è un tratto ricorrente. Scarse le interiezioni -in carattere con una tendenza generale delle tragedie di Seneca ${ }^{48}$ - che veicolano l'espressione dello stato d'animo del parlante in modo spontaneo e sono pertanto ritenute, nelle fonti letterarie in cui compaiono, prevalentemente come un tratto mimetico del parlato colloquiale. Allargando l'orizzonte alla struttura della conversazione ricordiamo la frequente realizzazione non standard di apertura e chiusura delle liti: accanto alle osservazioni sull'apertura -(1) e n.

${ }^{47}$ Una ricerca lessicale condotta sul database Packard Humanities Institute online Resource (PHI) sui lemmi della famiglia clamo (15 occorrenze) e strepo (10 occorrenze) per le tragedie di Seneca, l'Hercules Oetaeus e l'Octavia ha rilevato che i lemmi risultano impiegati principalmente ad indicare manifestazioni naturali, o rumori provocati da oggetti inanimati, o vociare indistinto di folla, il cui tratto comune sembra essere il volume alto. In sei casi per clamo (clamor: Sen., Tro. 678; Herc. O. 798; conclamo: Sen., Oed. 975; exclamo: Sen., Herc. F. 771; Oct. 332; proclamo: Sen., Med. 388) e in due per strepo (obstrepo: Sen., Herc. F. 1031; Sen., Ag. 289) i lemmi designano manifestazioni vocali di singoli, in tutti i casi volte ad esprimere emozioni negative quali ira e dolore; solo in quattro di questi (Sen., Herc. F. 771, 1031; Sen., Ag. 289; Oct. 332) si possono riconoscere manifestazioni (unilaterali) di aggressività verbale $\mathrm{i}$ cui protagonisti sono per lo più personaggi di statuto alto (rispettivamente Caronte, Anfitrione, Egisto ed Agrippina). Dallo spoglio delle occorrenze ricaviamo quindi qualche indicazione su casi in cui il volume alto della voce contraddistingue manifestazioni di emozioni negative ed aggressività verbale e si può suggerire che tale tratto comparisse anche nelle liti. Una conferma in proposito la si può leggere nell'occorrenza di Sen., Her. F. 172-173, in cui le liti (rabiosa ... / iurgia) sono presentate come tipiche di una piazza chiassosa (clamosi ... fori). Il tratto dell'eccesso emotivo (rabiosa) risulta quindi realizzato con manifestazioni vocali contrassegnate dal volume alto della voce (clamosi).

${ }^{48}$ O tumide ... timide ... regum tyranne (Sen., Tro. 301-303); O clarum trahens / stirpe nomine regia (359-360); agedum (Sen., Herc. F. 397); quaeso (Sen., Med. 514). Sullo studio delle interiezioni in latino v. Unceta Gómez 2012, 2016, 2017 (sulle tragedie di Seneca; in particolare su $o$ con gli appellativi v. ivi 140). 
9- notiamo che anche la chiusura viene per lo più decisa unilateralmente con un cambio di interlocutore, come si vede in (3), (7), (9), (11). Caratteristica comune di questi casi è l'avvitarsi della conversazione sulla rigida ripetizione delle posizioni dei parlanti: l'unica soluzione sembra essere quella radicale di uscire dalla conversazione, e si è suggerito che ad operare questa scelta sia il personaggio che non trova argomenti da opporre nel contraddittorio. Conferma di questa tendenza si può leggere anche nell'unico caso in cui la chiusura è negoziata, quello della Medea, in cui la protagonista finge una riconciliazione per ottenere la sua vendetta, e sposta quindi coscientemente l'interazione su un piano non aggressivo, anzi, orientato sull'avvicinamento al proprio interlocutore, cui ben si confà una chiusura concordata. Passando ad un piano narratologico, e prendendo in esame le funzioni che la lite può ricoprire nell'economia della trama, si è notato come ricorrente sia la valenza di caratterizzazione dei personaggi, in conformità al quadro emerso per le liti della commedia e del romanzo. L'unico caso in cui la lite sembra rappresentare anche un punto di svolta nella trama è ancora quello della Medea: la scoperta dell'amore paterno di Giasone indica a Medea il punto più doloroso in cui colpirlo. Si noti però che questo avviene in una fase della conversazione in cui l'aggressività della lite è scemata, quando Giasone si impegna a fornire a Medea il conforto che lei richiederà per mitigare la durezza dell'esilio. Non lo scambio di aggressività quindi, ma un momento di manifestazione di interesse per l'altro fornisce la soluzione per superare il contraddittorio. Anche per questo caso insomma si può argomentare che la conversazione conflittuale sia rappresentata come improduttiva, come un blocco dell'azione che non contribuisce allo sviluppo degli eventi.

L'analisi pragmatica delle liti in tragedia ha rilevato un comportamento linguistico in sostanziale continuità con quanto emerso nell'analisi delle liti di commedia e romanzo: per quanto riguarda la conversazione conflittuale quindi, le fonti letterarie concordano su un codice di comportamento comune. Le convenzioni orientate su comico e tragico dei diversi generi letterari condizionano la scelta dei protagonisti ed il registro lessicale; il maggior numero di attestazioni rinvenute nel più ampio corpus della commedia e del romanzo presenta una maggiore varietà di situazioni, comprese quelle in cui l'intento comico porta a sovraccaricare l'aggressività espressa. Di converso, il numero ridotto degli esempi di liti in tragedia è risultato caratterizzato da una spiccata omogeneità per quanto riguarda statuto dei personaggi e forme di impoli- 
teness. Fatte salve le differenze stilistiche che si sono osservate, e che si è proposto di ricondurre alle convenzioni di genere letterario, la resa sul piano pragmatico appare trasversalmente condivisa: espressione, si può suggerire, dell'atteggiamento culturale di quel segmento della società romana da cui i testi vengono prodotti e fruiti, e che tende ad associare l'aggressività verbale a valutazioni negative. Litigare non è risolutivo, e chi litiga viene caratterizzato come in preda all'ira, un turbamento emotivo che in alcuni casi si aggiunge a tratti già negativi ${ }^{49}$ e caratterizza modelli di comportamento non certo da imitare.

\section{BIBLIOGRAFIA}

Berger, Ł. 2016: «Introducing the First Topic Slot in Plautine Dialogues», Roczniki Humanistyczne 64, pp. 89-110.

Bettini, M. 2000: «Guardarsi in faccia a Roma: le parole dell'apparenza fisica nella cultura latina», in Bettini, M. (ed.), Le orecchie di Hermes. Studi di antropologia e letterature classiche, Torino, pp. 314-357.

Brown, P. e Levinson, S. $1987^{2}$ [1978]: Politeness. Some universals in language usage, Cambridge.

Calabrese, E. 2009: Il sistema della comunicazione nella Fedra di Seneca, Palermo.

Calabrese, E. 2010: «Modalità linguistiche e relazionali di costruzione dell'identità nell'Agamennone di Seneca», Dionysus ex machina 1, pp. 119-151.

Canter, H. V. 1925: Rhetorical Elements in the Tragedies of Seneca, Urbana.

Casamento, A. 1999: «Lumina orationis. L'uso delle sententiae nelle tragedie di Seneca», Studi italiani di filologia classica 17, pp. 123-132.

Costa, C. D. N. 1989: Medea. Oxford, New York.

Culpeper, J. 1996: «Towards an anatomy of impoliteness», Journal of Pragmatics 25, pp. 349-367.

Culpeper, J. 2011: Impoliteness. Using language to cause offence, Cambridge.

Culpeper, J. 2016: «Impoliteness strategies», in Capone, A. e Mey, J. L. (eds.), Interdisciplinary Studies in Pragmatics, Culture and Society, Cham, pp. 421-445.

Dingel, J. 1985: «Senecas Tragödien: Vorbilder und poetische Aspekte», in Haase, W. (ed.), Aufstieg und Niedergang der römischen Welt. Sprache und Literatur: $B d$. 32. 2. Literatur der julisch-claudischen und der flavischen Zeit, Berlino, pp. 1052-1099.

49 Si pensi all'arroganza rinfacciata da Agamennone a Pirro (Sen. Tro. 339; 349) o alla superbia che contraddistingue Lico (Sen., Herc. F. 384-385). 
Fantham, E. 1982: Seneca's “Troades”, Princeton (N.J.).

Ferri, R. 2008: «Politeness in Latin Comedy. Some Preliminary Thoughts», Materiali e Discussioni per l'Analisi dei Testi Classici 61, pp. 15-28.

Fitch, J. G. 1981: «Sense-Pauses and Relative Dating in Seneca, Sophocles and Shakespeare», American Journal of Philology 102, pp. 289-307.

Goffman, E. 1967: Interaction Ritual: Essays on face-to-face behaviour, Harmondsworth.

Goffman, E. 1981: Forms of talk, Filadelfia.

Goldberg, S. M. 2014: "Greek and Roman Elements in Senecan Tragedy», in Damschen, G. e Heil, A. (eds.), Brill's companion to Seneca: Philosopher and dramatist, Leida, pp. 639-652.

Heil, A. 2013: Die dramatische Zeit in Senecas Tragödien, Leida.

Hofmann, M. E. 1983: «Conversation openings in the Comedies of Plautus», in Pinkster, H. (ed.), Latin Linguistics and Linguistic Theory. Proceedings of the $1^{\text {st }}$ International Colloquium on Latin Linguistics, Amsterdam, pp. 217-226.

Iurescia, F. 2019: Credo iam ut solet iurgabit. Pragmatica della lite a Roma, Gottinga.

Iurescia, F. e Martin, G. 2019: «Closing conflicts. Conversational Strategies across Greek and Roman tragedies», Lingue e Linguaggi 31, pp. 233-254.

Kerbrat-Orecchioni, C. 1997: «A multilevel approach in the study of talk-in-interaction», Pragmatics 7, pp. 1-20.

Kerbrat-Orecchioni, C. 2004: «Introducing polylogue», Journal of Pragmatics 36, pp. $1-24$.

Keulen, A. J. 2001: L. Annaeus Seneca Troades. Introduction, Text and Commentary, Leida.

Kienpointner, M. 1997: «Varieties of rudeness. Types and functions of impolite utterances», Functions of Language 4, pp. 251-287.

Kunz, F. 1897: Sentenzen in Senecas Tragodien, Wiener-Neustadt.

Leumann, M. $1977^{5}$ [1926]: Lateinische Laut- und Formenlehre, Monaco.

Levinson, S. C. 1983: Pragmatics, Cambridge.

Nigris Mores, S. de. 1972: «Sugli aggettivi latini in -ax», Acme 25, pp. 263-313.

Orlandini, A. e Poccetti, P. 2010: «Stratégies discursives de non-interpellation et échecs de l'interpellation dans des dialogues latins», CORELA HS-8, Ottobre 2010, <http://corela.edel.univ-poitiers.fr/index.php?id=1045> (05/03/2019), doi: 10.4000/corela. 1045 .

Paschalis, M. 2010: «Cassandra and the passionate lucidity of furor in Seneca's Agamemnon», in Tsitsiridis, S. (ed.), Parachoregema: Studies on ancient theatre in honour of Professor Gregory M. Sifakis, Heraklion, pp. 209-228. 
Poccetti, P. 2010: "Greeting and farewell expressions as evidence for colloquial language: between literary and epigraphical texts», in Dickey, E. e Chahoud, A. (eds.), Colloquial and Literary Latin, Cambridge, pp. 100-126.

Ricottilli, L. 2013: «Strategie comunicative "a carambola” in Terenzio (Ph. 350-377; Andr., 459-497; 740-795)», Dionysus ex machina 4, pp. 133-145.

Risselada, R. 1993: Imperatives and other directive expressions in Latin. A study in the pragmatics of a dead language, Amsterdam.

Roesch, S. 2008: «Les débuts de dialogue dans la comedie et la tragedie latines», in Bureau, B. e Nicolas, C. (edd.), Commencer et Finir. Débuts et fins dans les littératures grecque, latine et néolatine. Actes du colloque organisé les 29 et 30 septembre 2006 par l'Université Jean Moulin-Lyon 3 et l'ENS-LSH, Lione, pp. 207-222.

Scolari, L. 2018: Beneficium e iniuria. Rappresentazioni del dono e dell'offesa nel De beneficiis di Seneca, Palermo.

Seidensticker, B. 1969: Die Gesprächsverdichtung in den Tragödien Senecas, Heidelberg.

Speyer, A. 2003: Kommunikationsstrukturen in Senecas Dramen. Eine pragmatisch-linguistische Analyse mit statistischer Auswertung als Grundlage neuer Ansätze zur Interpretation, Gottinga.

Steidle, W. 1941: «Zu Senecas Troerinnen», Philologus 94, pp. 266-284.

Tarrant, R. J. 1976: Agamemnon, Cambridge.

Tarrant, R. J. 1978: «Senecan Drama and its antecedents», Harvard Studies in Classical Philology 82, pp. 213-263.

Traina, A. $2011^{4}$ [1974]: Lo stile drammatico del filosofo Seneca, Bologna.

Unceta Gómez, L. 2012: «Cuando los sentimientos irrumpen: valores expresivos de las interjecciones primarias en las comedias de Plauto», in López Gregoris, R. (ed.), Estudios sobre teatro romano: el mundo de los sentimientos y su expresión, Saragozza, pp. 347-395.

Unceta Gómez, L. 2016: «Cuando los sentimientos irrumpen: análisis de las interjecciones secundarias en las comedias de Plauto», in Penas Ibáñez, A. e García-Hernández, B. (edd.), Semántica latina y románica. Unidades de significado conceptual y procedimental, Berna, pp. 213-241.

Unceta Gómez, L. 2017: «Cuando las emociones irrumpen: análisis comparativo del empleo de las interjecciones en las comedias de Terencio y las tragedias de Séneca», Onomazein 38, pp. 107-146.

Unceta Gómez, L. 2018: «Gli studi sulla (s)cortesia linguistica in latino. Possibilità di analisi e proposte per il futuro», Studi e Saggi Linguistici 56, pp. 9-37.

Vandersmissen, M. 2019: Discours des personnages féminins chez Sénèque: Approches logométriques et contrastives d'un corpus théâtral, Bruxelles. 
LITIGARE IN TRAGEDIA：PER UNA PRAGMATICA DEL CONFLITTO 283

Wollams, S. e Brown, M. 1979: The Total Handbook of Transactional Analysis, Englewood Cliffs (N.J.).

Zwierlein, O. 1986: L. Annaei Senecae, Tragoediae, Incertorum auctorum Hercules [Oetaeus], Octavia, Oxford.

Fecha de recepción de la primera versión del artículo: 18/04/2019

Fecha de aceptación: 20/05/2019

Fecha de recepción de la versión definitiva: 29/05/2019 